\title{
CRISPR/Cas9-targeted mutagenesis of Os8N3 in rice to confer resistance to Xanthomonas oryzae pv. oryzae
}

\author{
Young-Ah Kim ${ }^{1 \dagger}$, Hyeran Moon ${ }^{2+}$ and Chang-Jin Park ${ }^{1,2,3^{*}}$ (1)
}

\begin{abstract}
Background: Genome editing tools are important for functional genomics research and biotechnology applications. Recently, the clustered regularly interspaced short palindromic repeats (CRISPR)/CRISPR-associated protein-9 (Cas9) system for gene knockout has emerged as the most effective genome-editing tool. It has previously been reported that, in rice plants, knockdown of the Os8N3 gene resulted in enhanced resistance to Xanthomonas oryzae pv. oryzae (Xoo), while displaying abnormal pollen development.

Results: The CRISPR/Cas9 system was employed to knockout rice Os8N3, in order to confer enhanced resistance to $X_{0 O}$. Analysis of the genotypes and edited Os $8 \mathrm{~N}_{3}$ in $\mathrm{T}_{0}, \mathrm{~T}_{1}, \mathrm{~T}_{2}$, and $\mathrm{T}_{3}$ transgenic rice plants showed that the mutations were transmitted to subsequent generations, and homozygous mutants displayed significantly enhanced resistance to Xoo. Stable transmission of CRISPR/Cas9-mediated Os8N3 gene editing without the transferred DNA (T-DNA) was confirmed by segregation in the $T_{1}$ generation. With respect to many investigated agronomic traits including pollen development, there was no significant difference between homozygous mutants and non-transgenic control plants under greenhouse growth conditions.
\end{abstract}

Conclusion: Data from this study indicate that the CRISPR/Cas9-mediated Os8N3 edition can be successfully employed for non-transgenic crop improvements.

Keywords: CRISPR/Cas9, Disease resistance, Os8N3, Rice, xa13, Xanthomonas oryzae pv. oryzae

\section{Background}

Rice (Oryza sativa L.) is one of the most important cereal crops in the world, directly feeding more people than any other crop. Bacterial blight, caused by Xanthomonas oryzae pv. oryzae $(X o o)$, is a prevalent and destructive rice disease that causes serious production loss worldwide (Zhang and Wang 2013). Enhancing rice plants' resistance to Xoo is known to be an economical and effective approach for managing rice bacterial blight.

Xoo pathogenicity depends on a specific class of virulence factors, called transcription activator-like (TAL) effectors, which mimic plant transcriptional activators

\footnotetext{
* Correspondence: cjpark@sejong.ac.kr

${ }^{\dagger}$ Young-Ah Kim and Hyeran Moon contributed equally to this work.

'Department of Plant Biotechnology, Sejong University, Seoul 05006, South Korea

${ }^{2}$ Department of Molecular Biology, Sejong University, Seoul 05006, South

Korea

Full list of author information is available at the end of the article
}

(Hutin et al. 2015; Blanvillain-Baufume et al. 2017). The TAL effectors target the host nucleus, where they bind to specific promoter elements of the plant genes and activate their expression, reprogramming the plant transcriptome (Schornack et al. 2013). The genomes of Xanthomonas strains typically contain highly variable numbers of TAL effectors between Asian Xoo (15-26), African Xoo (8-10), and North-American Xoo (0) (Erkes et al. 2017). The rice genes targeted by TAL effectors have been identified as host disease-susceptibility genes, acting as major susceptibility factors during rice and Xoo interactions. In some cases, DNA polymorphisms in the so-called TAL effector binding elements (EBEs), located at the promoter region of the susceptibility gene, lead to no development of the disease (Yang et al. 2006; Hutin et al. 2015). Rice Os8N3 (also known as OsSWEET11), which belongs to the Sugar Will Eventually be Exported Transporters (SWEET) family of sugar transporters, represents one of the susceptibility genes induced by TAL effectors (Yang et al. 2006; 
Chen 2014). The expression of Os8N3 is induced by strains of Xoo carrying pthXo1, which encodes the TAL effector PthXo1 (Yang et al. 2006; Yuan et al. 2009). PthXo1 from Xoo strain PXO99 directly activates Os8N3 through recognition of TAL EBEs located at the promoter region of Os8N3 (Romer et al. 2010). The recessive resistance gene $x a 13$ occurs as a series of natural alleles of the susceptibility gene Os8N3 (Yang et al. 2006; Yuan et al. 2009). Although it has not been clearly demonstrated, Os8N3 is believed to remove toxic copper from xylem vessels where Xoo multiplies and spreads (Yuan et al. 2010), and make nutrients easily available to Xoo for its growth and virulence to cause disease (Chen et al. 2010; Chen et al. 2012).

Genome editing technologies enable precise modification of DNA sequences in vivo and promise a novel revolution in crop improvement (Sun et al. 2016; Feng et al. 2013). The clustered regularly interspaced short palindromic repeats (CRISPR)/CRISPR-associated protein-9 (Cas9) system has revolutionized genome editing and become widely popular because of its specificity, simplicity, and versatility. It allows targeted genome editing in organisms guided by a customizable small noncoding RNA called single guide RNA (sgRNA). Once susceptibility genes targeted by TAL effectors have been identified, the CRISPR/Cas9-mediated genome editing strategy can be employed to create a target mutation in the susceptibility genes. Although it was not edited by the CRISPR/ Cas9, Os11N3 (also known as OsSWEET14), the susceptibility gene targeted by AvrXa7 and PthXo3, has been edited by Transcription Activator-Like Effector Nucleases (TALENs) to create bacterial blight-resistant rice through disrupting the EBE site in the promoter region ( $\mathrm{Li}$ et al. 2012; Blanvillain-Baufume et al. 2017). It can also be applied to negative regulators of disease resistance that have been studied for the last decades (Grand et al. 2012; Wang et al. 2015; Chern et al. 2005). However, to date, only a few examples of improvement of disease resistance using the CRISPR/Cas9 approach have been reported (Wang et al. 2016; Pyott et al. 2016; Peng et al. 2017). For Os8N3, studies on its knockdown rice plants using the gene silencing system and promoter mutations reported that they showed enhanced resistance to Xoo while displaying abnormal pollen development (Yang et al. 2006; Chu et al. 2006). Recently, CRISPR/Cas9-mediated knockout of Os $8 \mathrm{~N} 3$ displayed decreased sucrose concentration in the embryo sacs and defective grain filling, suggesting that Os8N3 plays important role in sucrose transport during early stage of rice grain filling (Ma et al. 2017; Yang et al. 2018).

Here, the CRISPR/Cas9-target mutagenesis of Os8N3 in Kitaake, a Japonica rice cultivar, is reported. The homozygous mutant lines carrying edited Os8N3 displayed significantly enhanced resistance to Xoo with normal pollen development. It was possible to select resistant mutant lines not containing the transferred DNA (T-DNA) by segregation in the $\mathrm{T}_{1}$ generation.

\section{Results \\ Os8N3 in the rice cultivar Kitaake}

Os8N3 was originally isolated as a susceptibility gene from the rice cultivar Nipponbare (Yang et al. 2006) and later, the EBE in its promoter element bound and activated by TAL effector PthXo1 of PXO99 was determined experimentally (Romer et al. 2010). In this study, rice cultivar Kitaake was investigated to see if it also carries the EBE sequence in the Os8N3 promoter region. Using the Kitaake database (Li et al. 2017), the promoter sequence of the Os8N3 gene, ranging from $-1000 \mathrm{bp}$ to $-1 \mathrm{bp}$ relative to the ATG start codon, was analyzed (Fig. 1a). The putative TATA box (TATAAA) is located at -32 upstream of the transcription start site $(+1)$. The promoter region including PthXo1 EBE (TGCATC TCCCCCTACTGTACACCAC), ranging from $-80 \mathrm{bp}$ to - $56 \mathrm{bp}$ upstream of the transcription start site, displayed $100 \%$ identity to Nipponbare (Yang et al. 2006). After inoculation with strain PXO99, Kitaake displayed strong induction of Os8N3 two days after inoculation (DAI) (Fig. 1b) and long water-soaked lesions (approximately 13-14 cm) 12 DAI (Fig. 1c). These results suggest that Kitaake carries a functional susceptible gene Os $8 \mathrm{~N} 3$, whose expression is induced by PXO99 possessing the TAL effector PthXo1.

\section{CRISPR/Cas9 design for $x a 13 / 0 s 8 N 3$ editing}

In monocot plants, the rice U3 small nuclear RNA promoter (OsU3) is generally used to express sgRNA (Belhaj et al. 2013). Recently, the efficiency of mutations targeted by sgRNAs driven by different small nuclear RNA promoters including OsU3, OsU6a, OsU6b, and OsU6c, were compared in an Indica cultivar 93-11 (Ma et al. 2015b). OsU6a was slightly more efficient in driving genome editing than the other promoters. It has also been reported that U6 promoters derived from the target plants function better than heterologous U6 promoters (Sun et al. 2015). Therefore, it was decided to use the OsU6a promoter isolated from the Japonica cultivar Kitaake. The OsU6a promoter amplified from Kitaake contains five single-nucleotide substitutions and one 5-bp deletion compared with one from Indica cultivar 93-11 (Additional file 1: Figure S1). The Arabidopsis U6 promoter in the CRISPR/Cas9 vector, pHAtC (Kim et al. 2016), was replaced with the Kitaake OsU6a promoter, and the resulting OsU6a::pHAtC was used for rice CRISPR/Cas9-mediated target mutagenesis.

To design a CRISPR/Cas9 that targets the Os8N3 gene, a 20-bp nucleotide sequence $(x a 13 m)$ in the first exon of Os8N3 was chosen as the target site (Fig. 2a). The $x a 13 m$ 


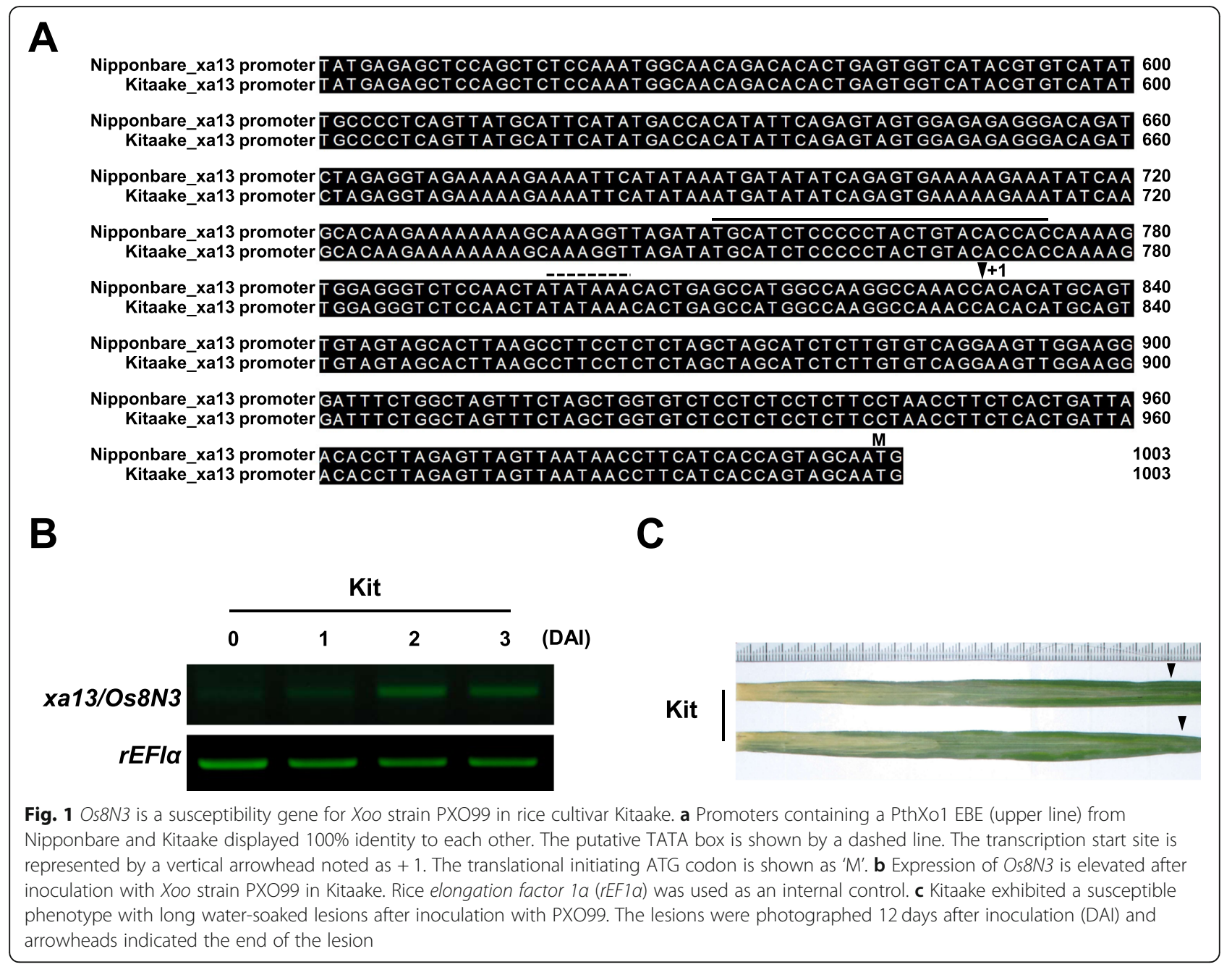

targeting sequence and protospacer adjacent motif (PAM) sequence are represented in red and in underlined lowercase letters, respectively. The predicted Cas 9 cleavage site (vertical arrowhead) in the coding region of the gene was 31 bp downstream from the ATG initiation codon. The recombinant binary plasmid, OsU6a::xa13m-sgRNA/ pHAtC, carrying $x a 13 m$-sgRNA targeting the Os8N3 gene under the control of the OsU6a promoter, was then constructed based on the OsU6a::pHAtC (Fig. 2b).

CRISPR/Cas9-mediated targeted mutagenesis of $x a 13 / 0$ s8N3 After Kitaake was transformed with OsU6a::xa13m$s g R N A / \mathrm{pHAtC}$ using Agrobacterium-mediated transformation, four independent transgenic Kitaake plants (OsU6a $x a 13 m / K i t \mathrm{~T}_{0}, 1 \mathrm{~A}, 2 \mathrm{~A}, 3 \mathrm{~A}$, and $4 \mathrm{~A}$ ) were generated. The putative transgenic plants were subjected to polymerase chain reaction (PCR)-based selection using the Cas9-specific primers, Cas9_RT_F and Cas9_RT-R (Fig. 2b), and all of them generated a Cas9-specific 400-bp amplicon (Fig. 3a). To further investigate CRISPR/Cas9targeted mutagenesis of $O s 8 \mathrm{~N} 3$, the target-containing amplicons obtained from all PCR-positive transgenic plants were directly sequenced and analyzed by decoding via the Degenerate Sequence Decoding method (Liu et al. 2015; Ma et al. 2015a). Rice plants are diploid with two copies of each gene, one copy on each chromosome of a chromosome pair. Therefore, when CRISPR/Cas9 is inserted into the genome and begins to function, one or both copies of the target gene Os8N3 can be cleaved and mutated, generating five possible genotypes in the transgenic plants: homozygote, biallele, heterozygote, chimera, and wild type (WT). In four $\mathrm{T}_{0}$ transgenic plants, there was only one homozygous mutation, 1-bp insertion $(+\mathrm{A})$, in $4 \mathrm{~A}$, whereas no target sequence changes could be detected in the other plants $\left(\mathrm{T}_{0}\right.$ in Table 1 and Additional file 2: Figure S2).

Inheritance of 0 s8N3 mutations and enhanced resistance to Xoo

To determine if and how the CRISPR/Cas9-targeted mutagenesis of Os8N3 by OsU6a::xa13m-sgRNA/pHAtC was transmitted to the next generation, all OsU6a 


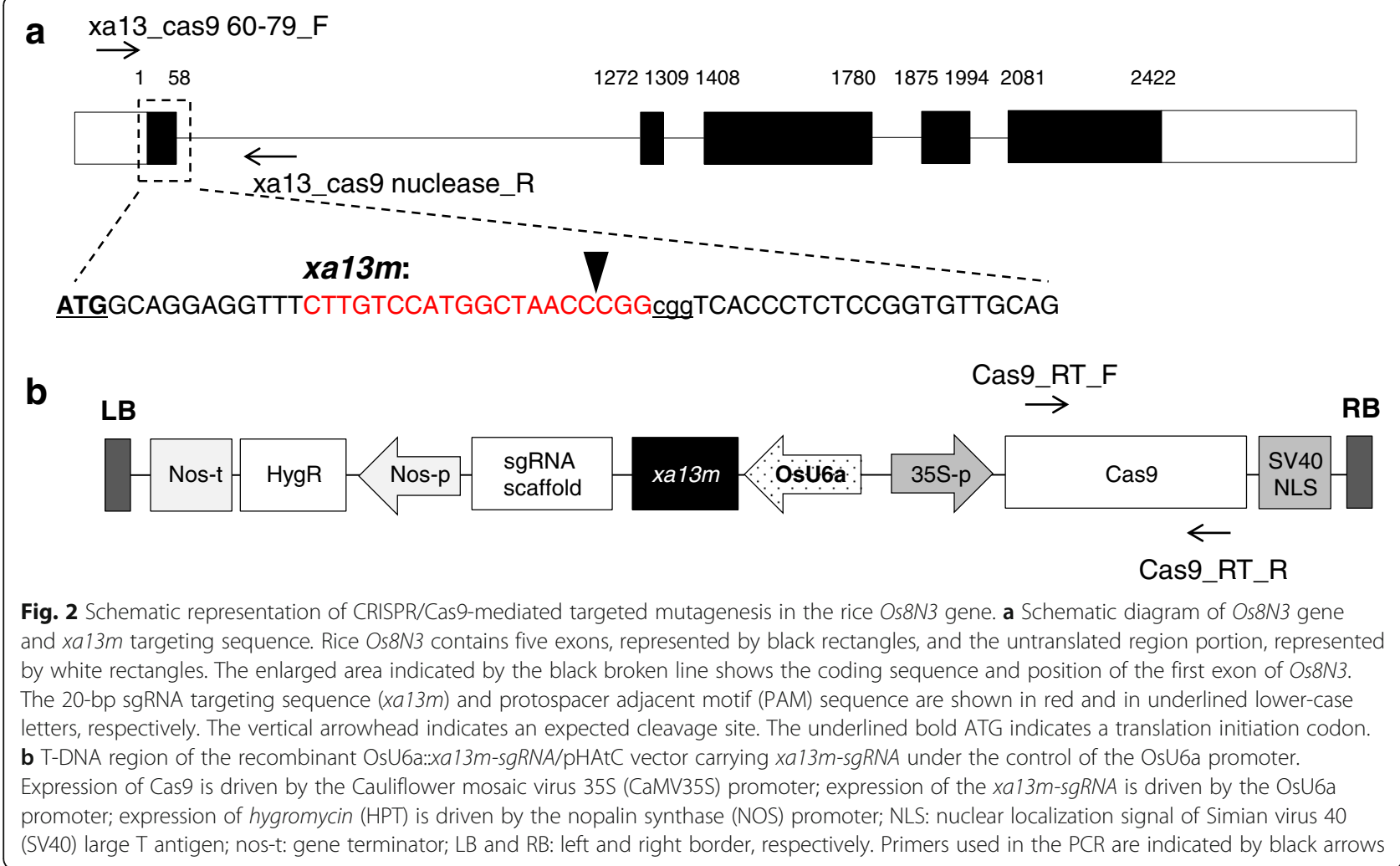

$x a 13 m / K i t \mathrm{~T}_{0}$ transgenic plants were self-pollinated and the targeted Os $8 N 3$ of some $\mathrm{T}_{1}$ transgenic plants was directly sequenced and analyzed (Fig. 3b, Table 1, and Additional file 3: Figure S3). The homozygous mutated $\mathrm{T}_{0}$ line $(4 \mathrm{~A})$ produced homozygous mutated $\mathrm{T}_{1}$ progeny (4A-1, 4A-2, and 4A-3) and did not display additional different mutations. There was no mutation observed in the sequenced $\mathrm{T}_{1}$ progenies of the WT $1 \mathrm{~A}$, and $2 \mathrm{~A}$ lines. However, new targeted sequence changes were detected in the $T_{1}$ progeny of the WT $3 \mathrm{~A}$ line. Previously, sequencing results indicated a putative WT genotype of the targeted Os $8 \mathrm{~N} 3$ in the $\mathrm{T}_{0} 3 \mathrm{~A}$ line, whereas three (3A-2, 3A-4, and 3A-6) out of the five sequenced $T_{1}$ progenies of the WT 3A line displayed a 1-bp insertion (Table 1): 3A-2 was homozygous; 3A-4 was bi-allelic; and $3 \mathrm{~A}-6$ was heterozygous.

To characterize the bacterial blight resistance phenotype of the mutant lines, $\mathrm{T}_{1}$ lines (progeny of OsU6a $x a 13 \mathrm{~m} / \mathrm{Kit} 1 \mathrm{~A}, 2 \mathrm{~A}, 3 \mathrm{~A}$, and $4 \mathrm{~A}$ ) with different types of allelic mutations were inoculated with PXO99 at the eight-week stage (Fig. 4a). Kitaake and transgenic Kitaake carrying Xa21 (XA21), driven by the ubiquitin promoter, were used as the susceptible and resistant control for PXO99, respectively (Park et al. 2010). As expected, while the XA21 plant was highly resistant, displaying short lesions, the inoculated leaves of the Kitaake plants developed long water-soaked lesions typical of bacterial blight disease. Homozygous (OsU6a $x a 13 m / K i t 3 \mathrm{~A}-2,4 \mathrm{~A}-1,4 \mathrm{~A}-2$, and $4 \mathrm{~A}-3$ ) and bi-allelic (3A-4) xa13 mutant plants displayed a robust resistance phenotype compared with heterozygous (3A-6) mutant and Kitaake control plants ( $\mathrm{T}_{1}$ in Table 1 and Fig. 4a). The differences were further evaluated by quantification of the lesion lengths and significance analysis using Tukey's HSD test (Fig. 4b). Homozygous and bi-allelic mutant plants displaying a resistance phenotype showed no significant differences in lesion lengths compared with the XA21 plants. These results indicated that the homozygous and bi-allelic mutant lines were significantly different from Kitaake and heterozygous mutant plants, and that CRISPR/Cas9-mediated mutagenesis in both Os8N3 alleles conferred robust resistance to PXO99.

To further investigate the inheritance of targeted mutations in later generations, the genotypes of several OsU6a $x a 13 m / K i t \mathrm{~T}_{2}$ plants were analyzed and inoculated with PXO99. New allelic mutation was detected in the $T_{2}$ progeny of WT 1A-5. Although all sequenced $\mathrm{T}_{0}$ and $\mathrm{T}_{1}$ generations of the $1 \mathrm{~A}$ line carry WT Os8N3, $\mathrm{T}_{2}$ progeny (1A-5-6) of the $1 \mathrm{~A}$ line displayed a heterozygous 1-bp insertion $(+\mathrm{T})$ mutation (Table 1 and Additional file 4: Figure S4). Heterozygous mutated 3A-6 $(+\mathrm{T})$ produced chimera 3A-6-1 with three distinct alleles detected at the target site, displaying additional different mutations $(+\mathrm{A})$. All $T_{1}$ plants derived from the homozygous $T_{0}$ mutant 


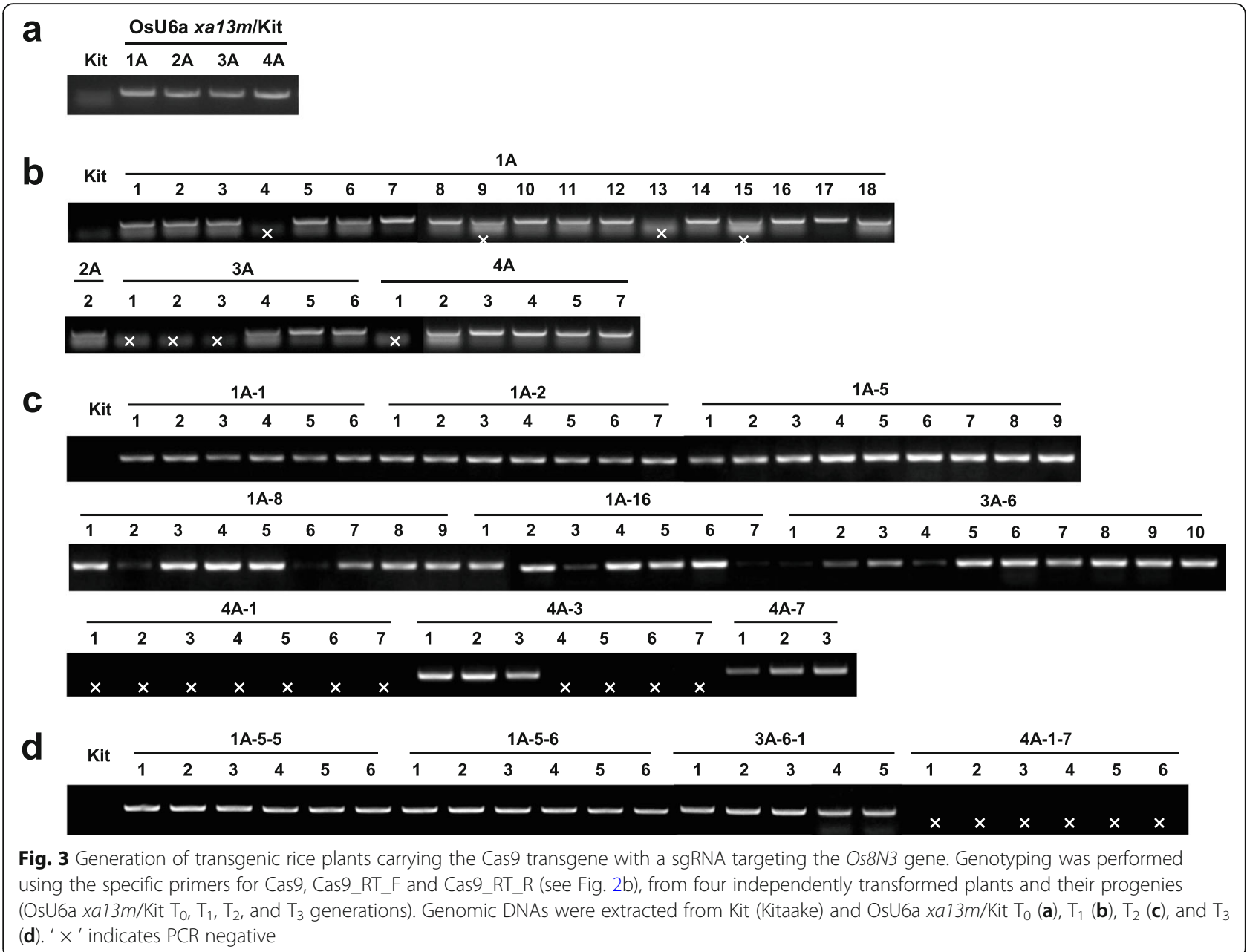

plant (4A) and $T_{2}$ plants derived from homozygous $T_{1}$ mutant plants (4A-1 and 4A-3) were homozygous for the same mutations (Table 1). All homozygous mutant lines (4A-1-6, 4A-1-7, 4A-3-3, and 4A-3-5) and chimera (3A-6-1) displayed significantly short lesion lengths (Fig. 5a and $\mathrm{b}$ ) and low bacterial populations compared with the heterozygous mutant (1A-5-6) and Kitaake plants (Fig. 5c). These results indicate that the mutations in these homozygous mutant lines and enhanced resistance to PXO99 were stably transmitted to the next generation.

\section{Main agronomic traits in xa13 mutants}

To determine whether mutations in the Os $8 N 3$ gene affect agronomic traits, two independent homozygous mutant lines $\left(\mathrm{T}_{3}\right)$ were analyzed by measuring their plant height, flag leaf length/width, the number of productive panicles, and panicle length (Table 2, Additional file 5: Figure S5 and Additional file 6: Figure S6). Tukey's HSD test indicated that the mutant lines displayed no significant difference to Kitaake, in terms of the investigated agronomic traits, under our greenhouse conditions.

Previously, Os8N3 knockdown transgenic plants displayed abnormal pollen development (Yang et al. 2006; $\mathrm{Chu}$ et al. 2006). To investigate whether Os8N3 knockout mutations affect pollen development, their pollen developments were assessed (Fig. 6). The phenotypical analysis showed that two independent homozygous $T_{3}$ mutant lines (3A-6-1-4 and 4A-1-7-6) exhibited normal golden yellow anthers (Fig. 6a). In addition, pollen grains from Kitaake and two independent homozygous $T_{3}$ mutant lines (3A-6-1-1 and 4A-1-7-1) were stained with iodine potassium iodide $\left(\mathrm{I}_{2}-\mathrm{KI}\right)$ (Fig. 6b). Dark-stained pollen grains (black in color) were considered viable and those that were lightly stained (yellow in color) were considered sterile. Homozygous mutants (3A-6-1-1 and 4A-1-7-1) displayed similar pollen viabilities to Kitaake, under our greenhouse conditions (Fig. 6c). The seed-setting rates and grain fillings were further analyzed in the Os8N3 knockout mutant lines (Additional file 7: Figure S7). Although, under greenhouse conditions, the 
Table 1 Transmission and segregation of CRISPR/Cas9-mediated target mutagenesis from $T_{0}, T_{1}, T_{2}$, and $T_{3}$ of the OsU6a xa13m/Kit transgenic plant. The recovered mutated alleles of the $x a 13 / 0$ s8N3 gene in the OsU6a $x a 13 m / K i t$ transgenic plant are shown below the Kitaake sequence. Nucleotide sequences at the target sites are shown in black capital letters and black dashes. PAM motifs are underlined. Red capital letters indicate the inserted nucleotide. The genotype of the mutation is indicated at the right of each sequence. WT indicates the nucleotide sequences identical to the Os8N3 gene in Kitaake plants. " + " indicates the insertion of the indicated number of nucleotides. No transgene: PCR negative for Cas9 gene; Transgenic: PCR positive for Cas9 gene; S: susceptible to PXO99; R: resistant to PXO99; Not available: inoculation data are not available

\begin{tabular}{|c|c|c|c|c|c|c|}
\hline & Line & Target sequence & Editing & Transgene & Zygosity & Phenotype \\
\hline & Kitaake & tttCTTGTCCATGGCTAACC-CGGcggtcacce & WT & No transgene & WT & $\mathrm{s}$ \\
\hline \multirow[t]{8}{*}{$\overline{T_{0}}$} & $1 \mathrm{~A}$ & tttCTTGTCCATGGCTAACC-CGGcggtcacce & WT & Transgenic & WT & $\mathrm{s}$ \\
\hline & & ttCTTGTCCATGGCTAACC-CGGcggtcaccc & WT & & & \\
\hline & $2 \mathrm{~A}$ & ttCTTGTCCATGGCTAACC-CGGcggtcacce & WT & Transgenic & WT & $\mathrm{s}$ \\
\hline & & tttCTTGTCCATGGCTAACC-CGGcggtcacce & WT & & & \\
\hline & $3 \mathrm{~A}$ & ttCTTGTCCATGGCTAACC-CGGcggtcaccc & WT & Transgenic & WT & $\mathrm{s}$ \\
\hline & & ttCTTGTCCATGGCTAACC-CGGcggtcacce & WT & & & \\
\hline & $4 \mathrm{~A}$ & tttCTTGTCCATGGCTAACCACGGcggtcacce & +1 & Transgenic & Homo & Not available \\
\hline & & tttCTTGTCCATGGCTAACCACGGcggtcacce & +1 & & & \\
\hline \multirow[t]{26}{*}{$\mathrm{T}_{1}$} & $1 \mathrm{~A}-1$ & tttCTTGTCCATGGCTAACC-CGGcggtcacec & WT & Transgenic & WT & $\mathrm{s}$ \\
\hline & & ttCTTGTCCATGGCTAACC-CGGcggtcacce & WT & & & \\
\hline & $1 \mathrm{~A}-5$ & ttCTTGTCCATGGCTAACC-CGGcggtcacce & WT & Transgenic & WT & $\mathrm{s}$ \\
\hline & & tttCTTGTCCATGGCTAACC-CGGcggtcacce & WT & & & \\
\hline & $1 \mathrm{~A}-8$ & tttCTTGTCCATGGCTAACC-CGGcggtcacce & WT & Transgenic & WT & $\mathrm{s}$ \\
\hline & & ttCTTGTCCATGGCTAACC-CGGcggtcacec & WT & & & \\
\hline & $1 \mathrm{~A}-16$ & tttCTTGTCCATGGCTAACC-CGGcggtcacce & WT & Transgenic & WT & $\mathrm{s}$ \\
\hline & & ttCTTGTCCATGGCTAACC-CGGeggtcacce & WT & & & \\
\hline & $2 \mathrm{~A}-2$ & tttCTTGTCCATGGCTAACC-CGGeggtcacec & WT & Transgenic & WT & $\mathrm{s}$ \\
\hline & & tttCTTGTCCATGGCTAACC-CGGeggtcacce & WT & & & \\
\hline & $3 \mathrm{~A}-2$ & tttCTTGTCCATGGCTAACCTCGGcggtcaccc & +1 & No transgene & Homo & $\mathrm{R}$ \\
\hline & & tttCTTGTCCATGGCTAACCTCGGcggtcacec & +1 & & & \\
\hline & $3 \mathrm{~A}-3$ & tttCTTGTCCATGGCTAACC-CGGcggtcacce & WT & No transgene & WT & $\mathrm{s}$ \\
\hline & & ttCTTGTCCATGGCTAACC-CGGcggtcacce & WT & & & \\
\hline & $3 \mathrm{~A}-4$ & tttCTTGTCCATGGCTAACCCCGGcggtcacce & +1 & Transgenic & Bi-allele & $\mathrm{R}$ \\
\hline & & ttCTTGTCCATGGCTAACCTCGGcggtcacce & +1 & & & \\
\hline & $3 \mathrm{~A}-5$ & ttCTTGTCCATGGCTAACC-CGGcggtcacec & WT & Transgenic & WT & Not available \\
\hline & & tttCTTGTCCATGGCTAACC-CGGcggtcacec & WT & & & \\
\hline & $3 \mathrm{~A}-6$ & ttCTTGTCCATGGCTAACC-CGGeggtcacce & WT & Transgenic & Hetero & $\mathrm{s}$ \\
\hline & & tttCTTGTCCATGGCTAACCTCGGcggtcaccc & +1 & & & \\
\hline & $4 \mathrm{~A}-1$ & tttCTTGTCCATGGCTAACCACGGcggtcacce & +1 & No transgene & Homo & $\mathrm{R}$ \\
\hline & & tttCTTGTCCATGGCTAACCACGGcggtcaccc & +1 & & & \\
\hline & $4 \mathrm{~A}-2$ & ttCTTGTCCATGGCTAACCACGGcggtcacce & +1 & Transgenic & Homo & $\mathrm{R}$ \\
\hline & & tttCTTGTCCATGGCTAACCACGGcggtcaccc & +1 & & & \\
\hline & $4 \mathrm{~A}-3$ & ttCTTGTCCATGGCTAACCACGGcggtcacce & +1 & Transgenic & Homo & $\mathrm{R}$ \\
\hline & & ttCTTGTCCATGGCTAACCACGGcggtcacce & +1 & & & \\
\hline \multirow[t]{21}{*}{$\overline{\mathrm{T}_{2}}$} & $1 \mathrm{~A}-5-5$ & tttCTTGTCCATGGCTAACC-CGGcggtcaccc & WT & Transgenic & WT & $\mathrm{s}$ \\
\hline & & ttCTTGTCCATGGCTAACC-CGGeggtcacce & WT & & & \\
\hline & $1 \mathrm{~A}-5-6$ & ttCTTGTCCATGGCTAACC-CGGcggtcacce & WT & Transgenic & Hetero & $\mathrm{s}$ \\
\hline & & ttCTTGTCCATGGCTAACCTCGGcggtcaccc & +1 & & & \\
\hline & $1 \mathrm{~A}-8-9$ & ttCTTGTCCATGGCTAACC-CGGeggtcacce & WT & Transgenic & WT & $\mathrm{s}$ \\
\hline & & ttCTTGTCCATGGCTAACC-CGGeggtcacce & WT & & & \\
\hline & $1 \mathrm{~A}-16-2$ & tttCTTGTCCATGGCTAACC-CGGeggtacec & WT & Transgenic & WT & $\mathrm{s}$ \\
\hline & & tttCTTGTCCATGGCTAACC-CGGcggtcacce & WT & & & \\
\hline & $3 \mathrm{~A}-6-1$ & ttCTTGTCCATGGCTAACC-CGGeggtcacce & WT & Transgenic & Chimera & $\mathrm{R}$ \\
\hline & & tttCTTGTCCATGGCTAACCACGGcggtcaccc & +1 & & & \\
\hline & & tttCTTGTCCATGGCTAACCTCGGcggtcaccc & +1 & & & \\
\hline & $3 \mathrm{~A}-6-3$ & tttCTTGTCCATGGCTAACC-CGGcggtcacce & WT & Transgenic & WT & $\mathrm{s}$ \\
\hline & & ttCTTGTCCATGGCTAACC-CGGcggtcacec & WT & & & \\
\hline & $4 \mathrm{~A}-1-6$ & tttCTTGTCCATGGCTAACCACGGcggtcacce & +1 & No transgene & Homo & $\mathrm{R}$ \\
\hline & & ttCTTGTCCATGGCTAACCACGGcggtcaccc & +1 & & & \\
\hline & $4 \mathrm{~A}-1-7$ & ttCTTGTCCATGGCTAACCACGGcggtcaccc & +1 & No transgene & Homo & $\mathrm{R}$ \\
\hline & & ttCTTGTCCATGGCTAACCACGGcggtcacce & +1 & & & \\
\hline & $4 \mathrm{~A}-3-3$ & tttCTTGTCCATGGCTAACCACGGcggtcaccc & +1 & Transgenic & Homo & $\mathrm{R}$ \\
\hline & & ttCTTGTCCATGGCTAACCACGGcggtcacce & +1 & & & \\
\hline & $4 \mathrm{~A}-3-5$ & ttCTTGTCCATGGCTAACCACGGcggtcaccc & +1 & No transgene & Homo & $\mathrm{R}$ \\
\hline & & tttCTTGTCCATGGCTAACCACGGcggtcacce & +1 & & & \\
\hline \multirow[t]{18}{*}{$\mathrm{T}_{3}$} & $1 \mathrm{~A}-5-5-1$ & tttCTTGTCCATGGCTAACC-CGGcggtcacce & WT & Transgenic & WT & Not available \\
\hline & & ttCTTGTCCATGGCTAACC-CGGcggtcacce & WT & & & \\
\hline & $1 \mathrm{~A}-5-5-3$ & ttCTTGTCCATGGCTAACC-CGGcggtcacce & WT & Transgenic & WT & Not available \\
\hline & & ttCTTGTCCATGGCTAACC-CGGeggtcacec & WT & & & \\
\hline & $1 \mathrm{~A}-5-5-4$ & tttCTTGTCCATGGCTAACC-CGGcggtcacec & WT & Transgenic & WT & Not available \\
\hline & & tttCTTGTCCATGGCTAACC-CGGeggtcacce & WT & & & \\
\hline & $1 \mathrm{~A}-5-5-5$ & ttCTTGTCCATGGCTAACC-CGGsggtcacec & WT & Transgenic & WT & Not available \\
\hline & & ttCTTGTCCATGGCTAACC-CGGcggtcacce & WT & & & \\
\hline & $1 \mathrm{~A}-5-5-6$ & tttCTTGTCCATGGCTAACC-CGGcggtcacce & WT & Transgenic & WT & Not available \\
\hline & & ttCTTGTCCATGGCTAACC-CGGeggtcacce & WT & & & \\
\hline & $3 \mathrm{~A}-6-1-1$ & tttCTTGTCCATGGCTAACCTCGGcggtcacce & +1 & Transgenic & Homo & Not available \\
\hline & & tttCTTGTCCATGGCTAACCTCGGcggtcaccc & +1 & & & \\
\hline & $3 \mathrm{~A}-6-1-2$ & ttCTTGTCCATGGCTAACCTCGGcggtcaccc & +1 & Transgenic & Homo & Not available \\
\hline & & ttCTTGTCCATGGCTAACCTCGGcggtcacce & +1 & & & \\
\hline & $3 \mathrm{~A}-6-1-3$ & ttCTTGTCCATGGCTAACCTCGGcggtcaccc & +1 & Transgenic & Homo & Not available \\
\hline & & tttCTTGTCCATGGCTAACCTCGGcggtcacce & +1 & & & \\
\hline & $3 \mathrm{~A}-6-1-4$ & tttCTTGTCCATGGCTAACCTCGGcggtcacce & +1 & Transgenic & Homo & Not available \\
\hline & & tttCTTGTCCATGGCTAACCTCGGcggtcacce & +1 & & & \\
\hline
\end{tabular}



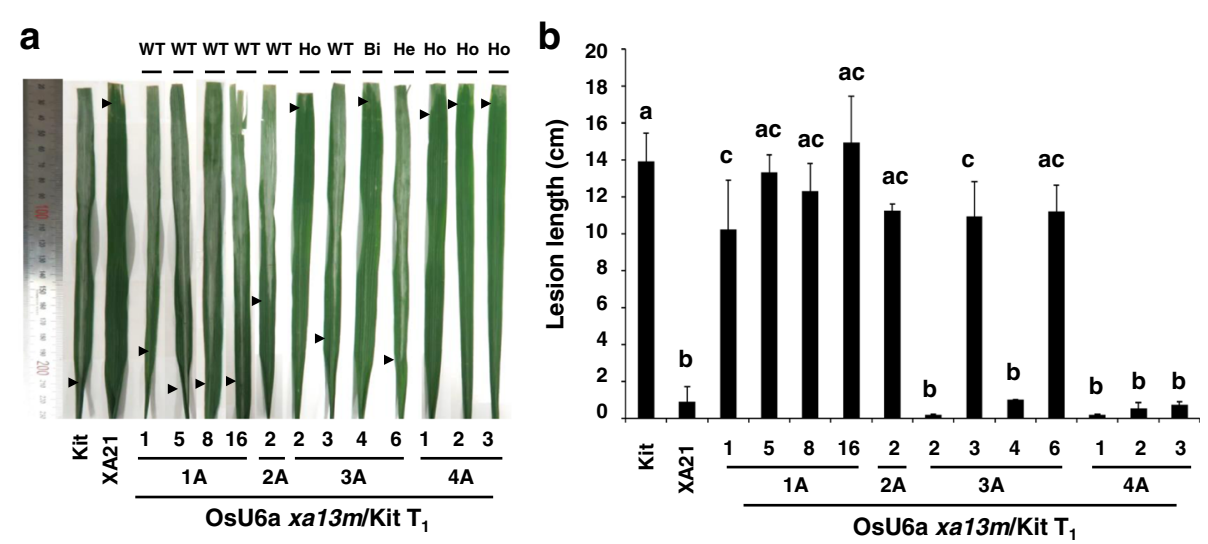

Fig. 4 CRISPR/Cas9-mediated mutagenesis in both OS8N3 alleles conferred enhanced resistance to Xoo. a Bacterial blight resistance phenotypes of the xa13 mutant rice lines $\left(T_{1}\right)$. Rice plants 12 DAl with Xoo. From left to right: Kitaake (Kit), transgenic line (XA21, 7A-8) carrying Xa21 driven by the ubiquitin promoter, and transgenic lines (OsU6a xa13m/Kit, $T_{1}$ ) carrying the OsU6a::xa13m-sgRNA/pHAtC construct. Arrowheads indicated the end of the lesion. WT; wild type: Ho; homozygous: Bi; bi-allelic: He; Heterozygous. b Lesion lengths measured 12 DAI in Kitaake, XA21, and OsU6a $x a 13 \mathrm{~m} / \mathrm{Kit} \mathrm{T}_{1}$. Error bars in the graph represent standard error of at least three leaves from each plant. Letters indicate a significant difference at $P<0.050$ by Tukey's HSD test

caryopses from two independent homozygous mutants (3A-6-1-1 and 4A-1-7-1) were slightly wrinkled as they matured (Additional file 7: Figure S7c), no significant alteration in the seed-setting rate was observed between progeny of two homozygous mutants (3A-6-1 and 4A1-7) and Kitaake plants (Additional file 7: Figure S7a and S7b).

\section{Selection of transgene-free mutant rice lines}

To select rice plants harboring the mutation in Os8N3 but without the T-DNA of the OsU6a::xa13m-sgRNA/ pHAtC construct, PCR and phenotypic analysis for the OsU6a $x a 13 m / K i t T_{0}, T_{1}$, and $\mathrm{T}_{2}$ plants was performed. Thirty-one segregating $\mathrm{T}_{1}$ plants were analyzed and six of them (19.35\%) did not generate a Cas9-specific amplicon from the T-DNA (Fig. 3b). Similarly, PCR analysis also failed to detect the T-DNA in 11 out of the $65 \mathrm{seg}$ regating $T_{2}$ plants $(16.92 \%)$ derived from nine $T_{1}$ plants (1A-1, 1A-2, 1A-5, 1A-8, 1A-16, 3A-6, 4A-1, 4A-3, and 4A-7) (Fig. 3c). Notably, the 4A-1 plant was a Cas9-free homozygous mutant harboring the desired $x a 13 / O s 8 N 3$ modifications (Fig. 3b and Fig. 4, and Additional file 3: Figure S3 and Additional file 4: Figure S4). None of the seven $\mathrm{T}_{2}$ plants derived from the $\mathrm{T}_{1}$ mutant plant $4 \mathrm{~A}-1$ generated the Cas9-specific amplicon (Fig. 3c). Two (4A-1-6 and 4A-1-7) out of the seven carried a 1-bp insertion $(+\mathrm{A})$ and displayed significantly enhanced resistance to PXO99 (Fig. 5), which has also been observed in their parent (4A-1) (Fig. 4). The $\mathrm{T}_{3}$ plant (4A-1-7-1) not generating the Cas9-specific amplicon carried the same Os8N3 modification observed in the $\mathrm{T}_{2}$ mutant plant 4A-1-7 (Fig. 3d and Additional file 4: Figure S4 and Additional file 5: Figure S5). These results indicate that T-DNA-free mutant plants carrying the desired gene modifications can be acquired through genetic segregation in $T_{1}, T_{2}$, and $T_{3}$ generations.

\section{Discussion}

The CRISPR/Cas9 system has been widely used to provide new avenues in crop improvements in rice, tomato, wheat, and maize (Xu et al. 2015; Feng et al. 2013; Wang et al. 2016; Ito et al. 2015; Wang et al. 2014; Zhou et al. 2014). In this study, OsU6a::pHAtC, which replaced the Arabidopsis U6 promoter in the pHAtC vector (Kim et al. 2016) with the OsU6a promoter of Kitaake, was constructed for rice CRISPR/Cas9-mediated target mutagenesis. Using the OsU6a::pHAtC, targeted mutagenesis in the recessive resistance gene, Os8N3, was generated.

One xa13 mutant line $4 \mathrm{~A}\left(\mathrm{~T}_{0}\right)$ from four independent transgenic OsU6a $x a 13 m / K i t$ plants carrying OsU6a:: xa13m-sgRNA/pHAtC was obtained. However, new targeted sequence changes were continuously detected in the transgenic OsU6a $x a 13 m / K i t$ plants in subsequent generations. For example, two additional independent mutant lines (progenies of $3 \mathrm{~A}$ and $1 \mathrm{~A}-5$ ) were identified in the $T_{1}$ and $T_{2}$ generations, respectively. Except for line $2 \mathrm{~A}$, which was lost in $\mathrm{T}_{1}$, all available lines in $\mathrm{T}_{2}$ were successfully mutated at the target sequence. Because the CRISPR/Cas9 system has been shown to be active in heterozygous and chimeric plants (Xu et al. 2015; Zhou et al. 2014), it is possible for the WT allele to be continuously modified in subsequent generations. Therefore, non-mutated transgenic plants, in which the OsU6a::xa13m-sgRNA/pHAtC construct remained active, continually cleaved the target site for generations, resulting in new mutations. Multiple mutations were also detected at the target site in the $T_{2}$ 


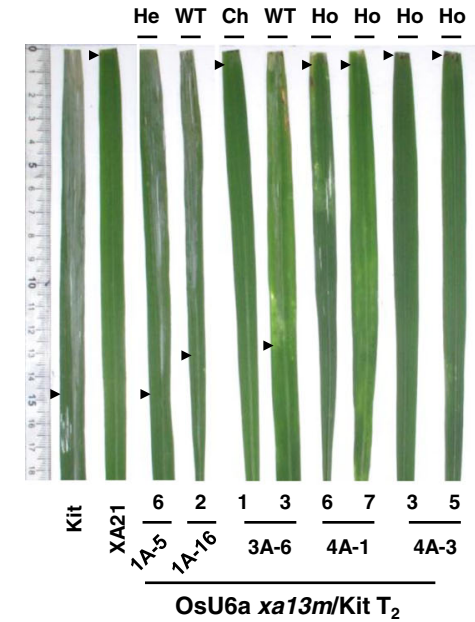

b

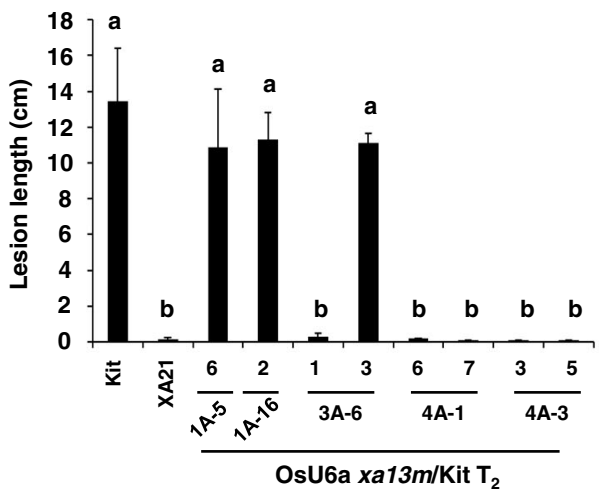

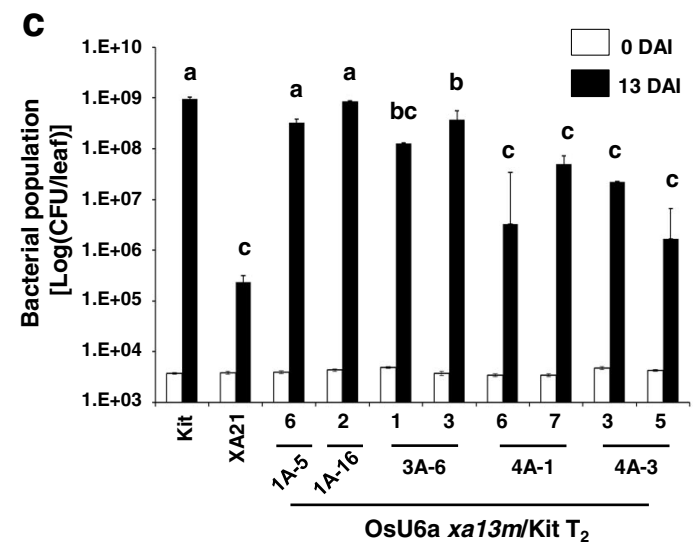

Fig. 5 Homozygous mutants in both Os8N3 alleles displayed enhanced resistance to Xoo. Transgenic Kitaake plants targeting xa13 (OsU6a xa13m/ $\mathrm{Kit}_{2}$ ) display enhanced resistance to $X_{00}$. a Inoculation results for mutant rice lines 12 DAl with Xoo. From left to right: Kitaake (Kit), transgenic line (XA21, 7A-8) carrying Xa21 driven by the ubiquitin promoter, and transgenic lines (OsU6a xa13m/Kit, $T_{2}$ ) carrying the OsU6a::xa13m-sgRNA/ pHAtc construct. Arrowheads indicated the end of the lesion. He; Heterozygous; WT; wild type: Ch; chimeric: Ho; homozygous. b Lesion lengths measured 12 DAl in Kitaake, XA21, and OsU6a xa13m/Kit T2. Error bars in the graph represent standard error of at least three leaves from each plant. Letters indicate a significant difference at $\mathrm{P}<0.050$ by Tukey's HSD test. c Bacterial population in Kitaake, XA21, and OsU6a xa13m/Kit T2 plants 0 and 12 DAl, determined by the number of CFU per inoculated leaf. Error bars represent standard deviation from at least three technical replicates. Letters indicate a significant difference at $\mathrm{P}<0.050$ by Tukey's HSD test

mutant plant 3A-6-1. Because 3A-6 was heterozygous, the presence of a chimeric mutation may result from delayed cleavage in the primary embryogenic cell of 3A-6-1. This chimeric mutation by the CRISPR/Cas9 system is likely a common phenomenon and has been reported in many plant species including rice $(\mathrm{Xu}$ et al.
2015; Feng et al. 2013; Wang et al. 2016), Arabidopsis (Feng et al. 2014), and tomato (Ito et al. 2015).

Regarding all examined agronomic traits, there was no significant difference between $\mathrm{T}_{3}$ homozygous mutants and Kitaake plants under greenhouse growth conditions. The homozygous mutant plants had a similar height, flag

Table 2 Analysis of the agronomic traits of $T_{3}$ mutant lines

\begin{tabular}{|c|c|c|c|c|c|}
\hline & Plant height (cm) & Flag leaf length $(\mathrm{cm})$ & Flag leaf width (mm) & No. of productive panicles & Panicle length $(\mathrm{cm})$ \\
\hline Kitaake & $69.8 \pm 4.1^{a}$ & $27.8 \pm 4.9^{a}$ & $11.7 \pm 0.9^{a}$ & $3.0 \pm 0.0^{a}$ & $11.1 \pm 2.1^{a}$ \\
\hline Progeny of 3A-6-1 & $65.6 \pm 6.4^{a}$ & $26.9 \pm 4.0^{a}$ & $12.7 \pm 0.5^{a}$ & $3.0 \pm 0.0^{a}$ & $12.1 \pm 1.4^{\mathrm{a}}$ \\
\hline Progeny of $4 \mathrm{~A}-1-7$ & $65.7 \pm 7.9^{a}$ & $29.1 \pm 4.8^{a}$ & $12.0 \pm 0.4^{a}$ & $2.5 \pm 1.1^{\mathrm{a}}$ & $12.0 \pm 2.2^{a}$ \\
\hline
\end{tabular}

The results shown are from more than three homozygous mutants of each mutant line, and are represented as the mean $\pm \mathrm{SE}$. The values marked with the same letter $\left({ }^{\mathrm{a}}\right)$ are non-significantly different $(P<0.050$, Tukey's HSD test) 


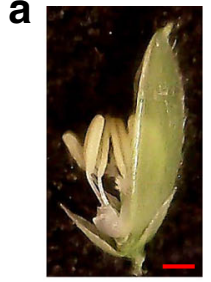

Kitaake

b

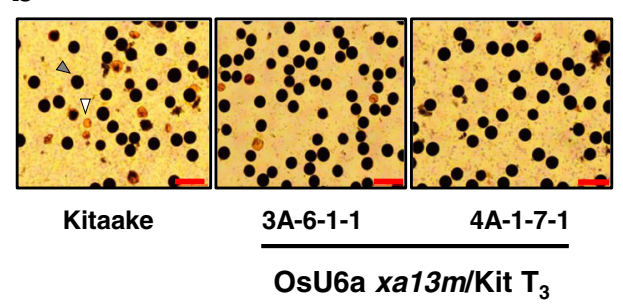

C

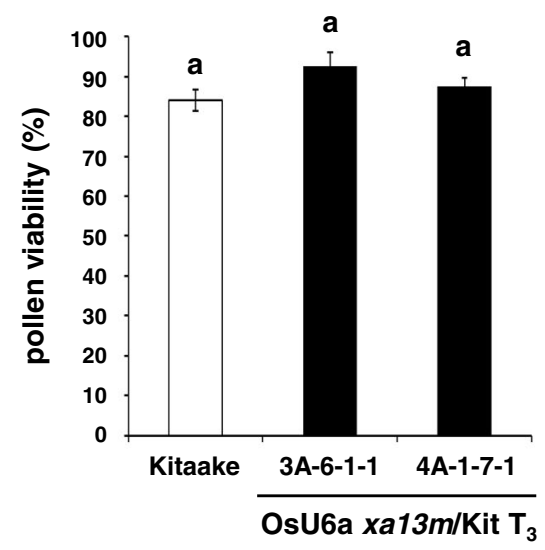

Fig. 6 Pollen viability of the homozygous xa13 mutants. a Anthers in mature spikelets of Kitaake, homozygous mutant ( $T_{3}$, 3A-6-1-4), and homozygous mutant ( $\left.T_{3}, 4 A-1-7-6\right)$. Scale bars, $1 \mathrm{~mm}$. b Representative images of pollen viability tests from Kitaake and homozygous mutants ( $T_{3}, 3 A-6-1-1$ and 4A-1-7-1). Viable pollen grains are stained dark (gray arrow) and sterile pollen grains are stained light yellow (white arrow). Scale bars, $100 \mu \mathrm{m}$. c Statistical analysis of pollen viability of Kitaake, homozygous mutants ( $T_{3}, 3 A-6-1-1$ and 4A-1-7-1) lines. Pollen viability percentage was calculated relative to the total pollen counted in three microscopic images

leaf length and width, number of productive panicles, panicle length, and pollen viability to Kitaake plants. It has been previously reported that Os8N3 is expressed at a high level in panicles and anthers during pollen development (Chu et al. 2006; Yang et al. 2006). Consistent with these observations, although detailed molecular mechanisms have not been elucidated, Os8N3-silenced rice plants displayed reduced fertility, and most pollen grains were defective (Chu et al. 2006; Yang et al. 2006). Therefore, Os8N3, conferring disease resistance by expressional loss-of-function in rice, has been considered an essential constituent for pollen development. However, in this study, homozygous mutants in both Os $8 \mathrm{~N} 3$ alleles were generated, and the mutations were stably transmitted to later generations, $T_{3}$. The homozygous $T_{3}$ mutant plants had normal pollen development, and most pollen grains were well preserved, in comparison with ones from Kitaake plants.

Thus far, it has been believed that Os8N3 plays roles in both copper and sugar transport, indicating its complex function in copper/sugar metabolism and signaling (Chen et al. 2010; Chen 2014; Yuan et al. 2010). However, no one dissected the molecular connection between Xoo resistance by copper/sugar metabolism and pollen development. Among the different in vivo functions of xa13/ Os8N3, knockout mutation, in particular, displayed enhanced resistance against Xoo without affecting pollen development. It is not yet understood why OsU6a $x a 13 m /$ Kit mutant lines did not display the sterile phenotype previously observed in Os8N3-knockdown rice plants (Chu et al. 2006; Yang et al. 2006). Because frameshift mutations of Os8N3 in OsU6a $x a 13 m /$ Kit lines are located at the very beginning of the Os8N3 polypeptide, it is very unlikely that the mutated polypeptide is functional. Lack of a functional Os8N3 protein in the mutant lines was also supported by a robust resistant phenotype of the homozygous mutant lines, but not heterozygous or Kitaake plants. Therefore, it is possible that there is a novel gene genetically compensating essential pollen development directly or indirectly in homozygous OsU6a $x a 13 m / K i t$ mutant lines. Genetic compensation was recently proposed to explain increasing numbers of studies revealing phenotypic differences between knockouts and knockdowns in plants (Gao et al. 2015; Braun et al. 2008; Chen et al. 2014) and animals (Young et al. 2009; De Souza et al. 2006; Daude et al. 2012; McJunkin et al. 2011; Law and Sargent 2014; Evers et al. 2016; Karakas et al. 2007; Morgens et al. 2016; Kok et al. 2015; Rossi et al. 2015). For example, similar to Os8N3, there have been studies on Arabidopsis auxinbinding protein 1 (ABP1) that revealed phenotypic differences between knockouts and knockdowns (Gao et al. 2015; Braun et al. 2008; Chen et al. 2014). Inducible $a b p 1$ knockdown lines showed defects in shoot and root growth, cell remodeling, or clathrin-mediated endocytosis of PIN auxin efflux carriers (Braun et al. 2008; Paque et al. 2014; Robert et al. 2010). However, abp1 knockout mutants generated by CRISPR/Cas 9 are indistinguishable from wild type plants at every developmental stage 
analyzed (Gao et al. 2015). Although one possible explanation for the difference is off-target effects of $A B P 1$ antisense RNA, it is not yet understood how independent abp1 knockdown lines, which generate fundamentally different approaches for functional down-regulation of the $A B P 1$ gene, display indistinguishable morphological defect phenotypes (Michalko et al. 2016). Recently, genetic compensation was studied in depth on zebrafish (Rossi et al. 2015). While knockdown of zebrafish EGF-like domain 7 (egfl7), an endothelial extracellular matrix gene, leads to severe vascular defects, most egfl7 mutants display no obvious defects (Rossi et al. 2015). Elastin microfibril interfacer (Emilin) genes were proposed as compensating genes in the edgl7 knockout mutants (Rossi et al. 2015). Supporting this hypothesis, Os8N3 mutants showed increased expressions of several SWEET genes such as OsSWEET3a, OsSWEET6b, OsSWEET13, and OsSWEET15 (Ma et al. 2017; Yang et al. 2018) and double mutants of Os8N3 and OSSWEET15 displayed much more wrinkled grain morphology, compared with single Os8N3 mutant (Yang et al. 2018). These reports suggest that some of SWEET genes are able to at least partially compensate for the lack of Os8N3. Currently, we are trying to identify candidate genes that compensate for $x a 13 / O s 8 N 3$ in the pollen development pathway without affecting Xoo resistance in homozygous mutant lines.

\section{Conclusions}

In summary, the CRISPR/Cas9 system was highly efficient in generating Os8N3 gene editing in rice. Mutant lines harboring the desired modification in Os $8 \mathrm{~N} 3$ but without the T-DNA of the OsU6a::xa13m-sgRNA/pHAtC were obtained. T-DNA-free homozygous mutant lines displayed significantly enhanced resistance to Xoo and normal pollen development. This study provides a successful example of improving bacterial blast resistance using CRISPR/Cas9 technology.

\section{Materials and methods}

\section{Plant and pathogen materials}

Rice cultivar Kitaake (Oryza sativa L. ssp. Japonica) was generously provided by Prof. Pamela Ronald (University of California Davis, USA). Rice plants in this study were maintained in the greenhouse facility at Sejong University in Korea. Xoo strain PXO99 was used in this study. PXO99 was cultured in peptone sucrose agar media (PSA: peptone $10.0 \mathrm{~g} / \mathrm{L}$, sucrose $1.0 \mathrm{~g} / \mathrm{L}$, L-glutamic acid $1.0 \mathrm{~g} / \mathrm{L}$, and agar $16.0 \mathrm{~g} / \mathrm{L}$ ) containing $15.0 \mathrm{mg} / \mathrm{L}$ cephalexin at $28^{\circ} \mathrm{C}$ for two days (Bai et al. 2000).

\section{Vector construction}

The Gateway ${ }^{\text {TI }}$ destination vector, pHAtC binary vector (Kim et al. 2016), was used to construct OsU6a::pHAtC carrying the OsU6a promoter to express sgRNA. A 472-bp
DNA fragment containing the OsU6a promoter (Ma et al. 2015c) was amplified from the genomic DNA of Kitaake using primers, EcoRI_OsU6a_F (5'-GGAATTCTTTTTTC CTGTAGTTTTCCCAC-3') and XhoI_OsU6a_R (5'GCTCGAGACACCTGCCTCCAATCCGGCAGCCAAG CCAGCACCC-3'). The PCR product was cloned into the pGEM ${ }^{\ominus}-\mathrm{T}$ Easy Vector according to the manufacturer's instructions (Promega, USA), and the insert was confirmed by Sanger sequencing. The OsU6a promoter was cut out from the $\mathrm{pGEM}^{\circ}-\mathrm{T}$ Easy Vector using $E c o R I+X h o I$ and cloned into the pHAtC, generating an OsU6a::pHAtC vector.

\section{Cloning of sgRNA expression vector}

The OsU6a::xa13m-sgRNA/pHAtC vector expressing sgRNA for $x a 13 / O s 8 N 3$ ( $x a 13 m$-sgRNA) was constructed according to the method previously described (Kim et al. 2016). Briefly, the target sequence (xa13m) for Os8N3 editing of Kitaake was designed by the CRISPR-RGEN Tools website (http://rgenome.ibs.re.kr) (Park et al. 2015). The sgRNA templates $(x a 13 m)$ for Os8N3 were annealed using two primers, 5 '-GATTGCTTGTCCATGGCTAACCCGG3' and 5' - AAACCCGGGTTAGCCATGGACAAGC-3', and cloned into Aarl-digested OsU6a::pHAtC. Construction of the sgRNA expression vector, OsU6a::xa13m$s g R N A / \mathrm{pHAtC}$, and its flanking sequences were confirmed by Sanger sequencing.

\section{Rice transformations}

Rice transformations were carried out as previously described (Chern et al. 2005). Agrobacterium tumefaciens strain LBA4404 was used to infect callus tissue induced from Kitaake seeds. Transformants carrying OsU6a: xa13m-sgRNA/pHAtC constructs were selected using hygromycin. Transgenic Kitaake plants overexpressing $x a 13 m$-sgRNA (OsU6a $x a 13 m / \mathrm{Kit}$ ) were confirmed by PCR using Cas9-specific primers, Cas9_RT_F (5'-CGAGCT GACCAAGGTGAAGT-3') and Cas9_RT_R (5'-CGTTGA TAAGCTTGCGGCTC-3').

\section{Expression}

For reverse transcription polymerase chain reaction (RTPCR) analysis of Cas 9 and sgxa13 transgenes, total RNA was extracted from fully expanded leaves of OsU6a $x a 13 m / K i t$ plants using TRIzol reagent (Invitrogen, USA). First-strand cDNA was synthesized using quantified RNA ( $5 \mu \mathrm{g}$ of total RNA). Expression of Cas9 was confirmed by RT-PCR using Cas9_RT_F and Cas9_RT_R. Meanwhile, the $r E F l a$ cDNA fragment was amplified as a control using specific primers, rEF1a1048F (5'-ACTGCCACACCTCC CACATTG-3') and rEF1a1552R (5' -CAACAGTCGAAG GGCAATAATAAGTC-3'). 


\section{Identification of mutant transgenic plants}

Rice genomic DNA was extracted from Kitaake leaves and transgenic OsU6a $x a 13 m / K i t$ plants. All transgenic hygromycin-resistant $\mathrm{T}_{0}$ plants were analyzed by PCR using the Cas9-specific primers, Cas9_RT_F and Cas9_ RT_R. Subsequently, the DNA fragment across the $x a 13$ target site was amplified from the genomic DNAs of all PCR-positive plants using xa13-specific primers, xa13 cas9 60-79_F (5' -TCTGGCTAGTTTCTAGCTGG-3') and xa13_cas9 nuclease_R (5'-TGCATGAGCTGAAG CTAGGG-3'). The PCR amplicons were then directly sequenced using primer xa13_cas9 60-79_F. The sequencing chromatograms with superimposed peaks of bi-allelic and heterozygous mutations were decoded using the Degenerate Sequence Decoding method (http://skl. scau.edu.cn/dsdecode/) (Liu et al. 2015; Ma et al. 2015a).

\section{Xoo inoculation and determination of bacterial populations}

For Xoo inoculation, Kitaake, XA21, and transgenic OsU6a $x a 13 m / K i t$ plants were grown in a greenhouse normally until they reached the eight-week stage, unless otherwise stated. PXO99 was used to inoculate rice plants using the scissors dip method (Song et al. 1995; Chern et al. 2005). For lesion length measurements, at least three inoculated leaves were measured to calculate the average and standard deviation 12 days after inoculation (DAI). Representative leaves were photographed 12 DAI. For Xoo colony counts from inoculated leaves 0 and $12 \mathrm{DAI}, 20 \mathrm{~cm}$ of leaf tissue from the top, including lesions and tissue showing no lesions, was ground up and resuspended in $10 \mathrm{ml}$ water to harvest bacteria. The extract was diluted accordingly and plated out on PSA plates containing $15.0 \mathrm{mg} / \mathrm{L}$ cephalexin. Plates were incubated at $28^{\circ} \mathrm{C}$ for two days, and then colony forming units (CFU) were counted. Statistical analysis was performed using Tukey's HSD test.

\section{Pollen viability tests}

Pollen viability was evaluated as previously described (Chhun et al. 2007). Before flowering, six anthers from Kitaake and transgenic OsU6a $x a 13 m / K i t$ plants were removed and crushed into a fine powder. Pollen grains were stained with $10 \mu \mathrm{I}_{2}-\mathrm{KI}$ solution $\left(1 \% \mathrm{I}_{2}, 3 \% \mathrm{KI}\right)$ and $1 \mu \mathrm{l}$ of stained pollen grains was harvested to observe fertile and infertile pollen under a light microscope. Dark-stained pollen grains were considered viable and the percentage of pollen viability was calculated relative to the total pollen counted in five microscopic images. Seed viability represents the percentage of spikelets that set seed per total number. Statistical analysis was performed using Tukey's HSD test.

\section{Additional files}

Additional file 1: Figure S1. Sequence comparison of OsU6a promoters from Japonica cultivar Kitaake and Indica cultivar 93-11. (PDF 72 kb)

Additional file 2: Figure S2. Sequencing chromatogram at the target site of Os8N3 in the CRISPR/Cas9-induced plants (OsU6a xa13m/Kit To). The vertical arrowhead indicates an expected cleavage site. (PDF 105 kb)

Additional file 3: Figure S3. Sequencing chromatogram at the target site of Os8N3 in the CRISPR/Cas9-induced plants (OsU6a xa13m/Kit T ). The vertical arrowhead indicates an expected cleavage site. (PDF $232 \mathrm{~kb}$ )

Additional file 4: Figure S4. Sequencing chromatogram at the target site of Os8N3 in the CRISPR/Cas9-induced plants (OsU6a xa13m/Kit T2). The vertical arrowhead indicates an expected cleavage site. (PDF 188 kb)

Additional file 5: Figure S5. Sequencing chromatogram at the target site of Os8N3 in the CRISPR/Cas9-induced plants (OsU6a xa13m/Kit T3). The vertical arrowhead indicates an expected cleavage site. (PDF 196 kb)

Additional file 6 Figure S6. Gross morphology of Kitaake and two homozygous Os8N3 mutant lines, $T_{3}$ progeny of $3 A-6-1$ and $4 A-1-7$. (PDF $72 \mathrm{~kb})$

Additional file 7: Figure S7. Seed-setting rates of homozygous xa13 mutants. a Representative panicles from Kitaake, homozygous mutant $\left(T_{3}\right.$, $3 A-6-1-2)$, and homozygous mutant $\left(T_{3}, 4 A-1-7-4\right)$. b Seed-setting rates of Kitaake, homozygous mutant (progeny of $3 A-6-1)$, and homozygous mutant (progeny of 4A-1-7). c Mature caryopses of Kitaake, homozygous mutant $\left(T_{3}, 3 A-6-1-1\right)$, and homozygous mutant $\left(T_{3}, 4 A-1-7-1\right)$. Scale bars, $2.5 \mathrm{~mm}$. (PDF $78 \mathrm{~kb}$ )

\section{Abbreviations}

CRISPR/Cas9: Clustered regularly interspaced short palindromic repeats/ CRISPR-associated protein-9 nuclease; EBE: Effector binding element; TAL effector: Transcription activator-like effector; Xoo PR6: Xanthomonas oryzae pv. oryzae Philippine Race 6

\section{Acknowledgments}

We thank Hyesu Jo for technical assistance in generating transgenic plants.

\section{Authors' contributions}

YAK, HM and CJP conceived and designed the experiments. YAK and HM performed the experiments and analyzed the data. YAK, HM, and CJP wrote the manuscript. All authors read and approved the final manuscript.

\section{Funding}

This work was supported by the Basic Research Program of National Research Foundation of Korea (NRF-2017R1D1A1B03032215), funded by the Ministry of Science and ICT.

\section{Availability of data and materials}

All data generated or analysed during this study are included in this published article and its supplementary information files.

Ethics approval and consent to participate

Not applicable.

\section{Consent for publication}

The manuscript has been approved by all authors.

\section{Competing interests}

The authors declare that they have no competing interests.

\section{Author details}

'Department of Plant Biotechnology, Sejong University, Seoul 05006, South Korea. ${ }^{2}$ Department of Molecular Biology, Sejong University, Seoul 05006, South Korea. ${ }^{3}$ Plant Engineering Research Institute, Sejong University, Seoul 05006, South Korea. 
Received: 1 March 2019 Accepted: 13 August 2019 Published online: 24 August 2019

\section{References}

Bai J, Choi SH, Ponciano G, Leung H, Leach JE (2000) Xanthomonas oryzae pv. Oryzae avirulence genes contribute differently and specifically to pathogen aggressiveness. Mol Plant-Microbe Interact 13(12):1322-1329. https://doi. org/10.1094/MPMI.2000.13.12.1322

Belhaj K, Chaparro-Garcia A, Kamoun S, Nekrasov V (2013) Plant genome editing made easy: targeted mutagenesis in model and crop plants using the CRISPR/ Cas system. Plant Methods 9(1):39. https://doi.org/10.1186/1746-4811-9-39

Blanvillain-Baufume S, Reschke M, Sole M, Auguy F, Doucoure H, Szurek B, Meynard D, Portefaix M, Cunnac S, Guiderdoni E, Boch J, Koebnik R (2017) Targeted promoter editing for rice resistance to Xanthomonas oryzae pv. oryzae reveals differential activities for SWEET14-inducing TAL effectors. Plant Biotechnol J 15(3):306-317. https://doi.org/10.1111/pbi.12613

Braun N, Wyrzykowska J, Muller P, David K, Couch D, Perrot-Rechenmann C, Fleming AJ (2008) Conditional repression of AUXIN BINDING PROTEIN1 reveals that it coordinates cell division and cell expansion during postembryonic shoot development in Arabidopsis and tobacco. Plant Cell 20(10):2746-2762. https://doi.org/10.1105/tpc.108.059048

Chen LQ (2014) SWEET sugar transporters for phloem transport and pathogen nutrition. New Phytol 201(4):1150-1155

Chen LQ, Hou BH, Lalonde S, Takanaga H, Hartung ML, Qu XQ, Guo WJ, Kim $J G$, Underwood W, Chaudhuri B, Chermak D, Antony G, White FF, Somerville SC, Mudgett MB, Frommer WB (2010) Sugar transporters for intercellular exchange and nutrition of pathogens. Nature 468(7323):527532. https://doi.org/10.1038/nature09606

Chen LQ, Qu XQ, Hou BH, Sosso D, Osorio S, Fernie AR, Frommer WB (2012) Sucrose efflux mediated by SWEET proteins as a key step for phloem transport. Science 335(6065):207-211. https:/doi.org/10.1126/science.1213351

Chen X, Grandont L, Li H, Hauschild R, Paque S, Abuzeineh A, Rakusova H, Benkova E, Perrot-Rechenmann C, Friml J (2014) Inhibition of cell expansion by rapid ABP1-mediated auxin effect on microtubules. Nature 516(7529):9093. https://doi.org/10.1038/nature13889

Chern M, Canlas PE, Fitzgerald HA, Ronald PC (2005) Rice NRR, a negative regulator of disease resistance, interacts with Arabidopsis NPR1 and rice NH1. Plant J 43(5):623-635. https://doi.org/10.1111/j.1365-313X.2005.02485.x

Chhun T, Aya K, Asano K, Yamamoto E, Morinaka Y, Watanabe M, Kitano H, Ashikari M, Matsuoka M, Ueguchi-Tanaka M (2007) Gibberellin regulates pollen viability and pollen tube growth in rice. Plant Cell 19(12):3876-3888. https://doi.org/10.1105/tpc.107.054759

Chu Z, Yuan M, Yao J, Ge X, Yuan B, Xu C, Li X, Fu B, Li Z, Bennetzen JL, Zhang Q, Wang $S$ (2006) Promoter mutations of an essential gene for pollen development result in disease resistance in rice. Genes Dev 20(10):12501255. https://doi.org/10.1101/gad.1416306

Daude N, Wohlgemuth S, Brown R, Pitstick R, Gapeshina H, Yang J, Carlson GA, Westaway D (2012) Knockout of the prion protein (PrP)-like Sprn gene does not produce embryonic lethality in combination with PrP(C)-deficiency. Proc Natl Acad Sci U S A 109(23):9035-9040. https://doi.org/10.1073/pnas.1202130109

De Souza AT, Dai X, Spencer AG, Reppen T, Menzie A, Roesch PL, He Y, Caguyong MJ, Bloomer S, Herweijer H, Wolff JA, Hagstrom JE, Lewis DL, Linsley PS, Ulrich RG (2006) Transcriptional and phenotypic comparisons of Ppara knockout and siRNA knockdown mice. Nucleic Acids Res 34(16):44864494. https://doi.org/10.1093/nar/gkl609

Erkes A, Reschke M, Boch J, Grau J (2017) Evolution of transcription activator-like effectors in Xanthomonas oryzae. Genome Biol Evol 9(6):1599-1615. https://doi.org/10.1093/gbe/evx108

Evers B, Jastrzebski K, Heijmans JP, Grernrum W, Beijersbergen RL, Bernards R (2016) CRISPR knockout screening outperforms shRNA and CRISPRi in identifying essential genes. Nat Biotechnol 34(6):631-633. https://doi.org/10.1038/nbt.3536

Feng Z, Mao Y, Xu N, Zhang B, Wei P, Yang DL, Wang Z, Zhang Z, Zheng R, Yang L, Zeng L, Liu X, Zhu JK (2014) Multigeneration analysis reveals the inheritance, specificity, and patterns of CRISPR/Cas-induced gene modifications in Arabidopsis. Proc Natl Acad Sci U S A 111(12):4632-4637. https://doi.org/10.1073/pnas.1400822111

Feng Z, Zhang B, Ding W, Liu X, Yang DL, Wei P, Cao F, Zhu S, Zhang F, Mao Y, Zhu JK (2013) Efficient genome editing in plants using a CRISPR/Cas system. Cell Res 23(10):1229-1232. https://doi.org/10.1038/cr.2013.114
Gao Y, Zhang Y, Zhang D, Dai X, Estelle M, Zhao Y (2015) Auxin binding protein 1 (ABP1) is not required for either auxin signaling or Arabidopsis development. Proc Natl Acad Sci U S A 112(7):2275-2280. https://doi. org/10.1073/pnas.1500365112

Grand X, Espinoza R, Michel C, Cros S, Chalvon V, Jacobs J, Morel JB (2012) Identification of positive and negative regulators of disease resistance to rice blast fungus using constitutive gene expression patterns. Plant Biotechnol J 10(7):840-850. https://doi.org/10.1111/j.1467-7652.2012.00703.x

Hutin M, Perez-Quintero AL, Lopez C, Szurek B (2015) MorTAL Kombat: the story of defense against TAL effectors through loss-of-susceptibility. Front Plant Sci 6:535. https://doi.org/10.3389/fpls.2015.00535

Ito Y, Nishizawa-Yokoi A, Endo M, Mikami M, Toki S (2015) CRISPR/Cas9-mediated mutagenesis of the RIN locus that regulates tomato fruit ripening. Biochem Biophys Res Commun 467(1):76-82. https://doi.org/10.1016/j.bbrc.2015.09.117

Karakas B, Weeraratna AT, Abukhdeir AM, Konishi H, Gustin JP, Vitolo MI, Bachman KE, Park BH (2007) P21 gene knock down does not identify genetic effectors seen with gene knock out. Cancer Biol Ther 6(7):1025-1030

Kim H, Kim ST, Ryu J, Choi MK, Kweon J, Kang BC, Ahn HM, Bae S, Kim J, Kim JS, Kim SG (2016) A simple, flexible and high-throughput cloning system for plant genome editing via CRISPR-Cas system. J Integr Plant Biol 58(8):705712. https://doi.org/10.1111/jipb.12474

Kok FO, Shin M, Ni CW, Gupta A, Grosse AS, van Impel A, Kirchmaier BC, Peterson-Maduro J, Kourkoulis G, Male I, DeSantis DF, Sheppard-Tindell S, Ebarasi L, Betsholtz C, Schulte-Merker S, Wolfe SA, Lawson ND (2015) Reverse genetic screening reveals poor correlation between morpholinoinduced and mutant phenotypes in zebrafish. Dev Cell 32(1):97-108. https://doi.org/10.1016/j.devcel.2014.11.018

Law SH, Sargent TD (2014) The serine-threonine protein kinase PAK4 is dispensable in zebrafish: identification of a morpholino-generated pseudophenotype. PLoS One 9(6):e100268. https://doi.org/10.1371/journal.pone.0100268

Li G, Jain R, Chern M, Pham NT, Martin JA, Wei T, Schackwitz WS, Lipzen AM, Duong PQ, Jones KC, Jiang L, Ruan D, Bauer D, Peng Y, Barry KW, Schmutz J, Ronald PC (2017) The sequences of 1504 mutants in the model Rice variety Kitaake facilitate rapid functional genomic studies. Plant Cell 29(6):1218-1231. https://doi.org/10.1105/tpc.17.00154

Li T, Liu B, Spalding MH, Weeks DP, Yang B (2012) High-efficiency TALEN-based gene editing produces disease-resistant rice. Nat Biotechnol 30(5):390-392. https://doi.org/10.1038/nbt.2199

Liu W, Xie X, Ma X, Li J, Chen J, Liu YG (2015) DSDecode: a web-based tool for decoding of sequencing chromatograms for genotyping of targeted mutations. Mol Plant 8(9):1431-1433. https://doi.org/10.1016/j.molp.2015.05.009

Ma L, Zhang D, Miao Q, Yang J, Xuan Y, Hu Y (2017) Essential role of sugar transporter OsSWEET11 during the early stage of Rice grain filling. Plant Cell Physiol 58(5):863-873. https://doi.org/10.1093/pcp/pcx040

Ma X, Chen L, Zhu Q, Chen Y, Liu YG (2015a) Rapid decoding of sequencespecific nuclease-induced heterozygous and Biallelic mutations by direct sequencing of PCR products. Mol Plant 8(8):1285-1287. https://doi.org/10.1016/j.molp.2015.02.012

Ma X, Zhang Q, Zhu Q, Liu W, Chen Y, Qiu R, Wang B, Yang Z, Li H, Lin Y, Xie Y, Shen R, Chen S, Wang Z, Chen Y, Guo J, Chen L, Zhao X, Dong Z, Liu YG (2015b) A robust CRISPR/Cas9 system for convenient, high-efficiency multiplex genome editing in monocot and dicot plants. Mol Plant 8(8):12741284. https://doi.org/10.1016/j.molp.2015.04.007

McJunkin K, Mazurek A, Premsrirut PK, Zuber J, Dow LE, Simon J, Stillman B, Lowe SW (2011) Reversible suppression of an essential gene in adult mice using transgenic RNA interference. Proc Natl Acad Sci U S A 108(17):7113-7118. https://doi.org/10.1073/pnas.1104097108

Michalko J, Glanc M, Perrot-Rechenmann C, Friml J (2016) Strong morphological defects in conditional Arabidopsis abp1 knock-down mutants generated in absence of functional ABP1 protein. F1000Res 5:86. https://doi.org/10.12688/f1000research.7654.1

Morgens DW, Deans RM, Li A, Bassik MC (2016) Systematic comparison of CRISPR/Cas9 and RNAi screens for essential genes. Nat Biotechnol 34(6): 634-636. https://doi.org/10.1038/nbt.3567

Paque S, Mouille G, Grandont L, Alabadi D, Gaertner C, Goyallon A, Muller P, Primard-Brisset C, Sormani R, Blazquez MA, Perrot-Rechenmann C (2014) AUXIN BINDING PROTEIN1 links cell wall remodeling, auxin signaling, and cell expansion in arabidopsis. Plant Cell 26(1):280-295. https://doi.org/10.1105/tpc.113.120048

Park CJ, Lee SW, Chern M, Sharma R, Canlas PE, Song MY, Jeon JS, Ronald PC (2010) Ectopic expression of rice Xa21 overcomes developmentally 
controlled resistance to Xanthomonas oryzae pv. oryzae. Plant Sci 179(5): 466-471. https://doi.org/10.1016/.jplantsci.2010.07.008

Park J, Bae S, Kim JS (2015) Cas-designer: a web-based tool for choice of CRISPRCas9 target sites. Bioinformatics (Oxford, England) 31(24):4014-4016. https://doi.org/10.1093/bioinformatics/btv537

Peng A, Chen S, Lei T, Xu L, He Y, Wu L, Yao L, Zou X (2017) Engineering cankerresistant plants through CRISPR/Cas9-targeted editing of the susceptibility gene CsLOB1 promoter in citrus. Plant Biotechnol J 15(12):1509-1519. https://doi.org/10.1111/pbi.12733

Pyott DE, Sheehan E, Molnar A (2016) Engineering of CRISPR/Cas9-mediated potyvirus resistance in transgene-free Arabidopsis plants. Mol Plant Pathol 17(8):1276-1288. https://doi.org/10.1111/mpp.12417

Robert S, Kleine-Vehn J, Barbez E, Sauer M, Paciorek T, Baster P, Vanneste S, Zhang J, Simon S, Covanova M, Hayashi K, Dhonukshe P, Yang Z, Bednarek SY, Jones AM, Luschnig C, Aniento F, Zazimalova E, Friml J (2010) ABP1 mediates auxin inhibition of clathrin-dependent endocytosis in Arabidopsis. Cell 143(1):111-121. https://doi.org/10.1016/j.cell.2010.09.027

Romer P, Recht S, Strauss T, Elsaesser J, Schornack S, Boch J, Wang S, Lahaye T (2010) Promoter elements of rice susceptibility genes are bound and activated by specific TAL effectors from the bacterial blight pathogen, Xanthomonas oryzae pv. oryzae. New Phytol 187(4):1048-1057. https://doi.org/10.1111/j.1469-8137.2010.03217.x

Rossi A, Kontarakis Z, Gerri C, Nolte H, Holper S, Kruger M, Stainier DY (2015) Genetic compensation induced by deleterious mutations but not gene knockdowns. Nature 524(7564):230-233. https://doi.org/10.1038/nature14580

Schornack S, Moscou MJ, Ward ER, Horvath DM (2013) Engineering plant disease resistance based on TAL effectors. Annu Rev Phytopathol 51:383-406. https:// doi.org/10.1146/annurev-phyto-082712-102255

Song WY, Wang GL, Chen LL, Kim HS, Pi LY, Holsten T, Gardner J, Wang B, Zhai WX, Zhu LH, Fauquet C, Ronald P (1995) A receptor kinase-like protein encoded by the rice disease resistance gene, Xa21. Science 270(5243):1804-1806

Sun X, Hu Z, Chen R, Jiang Q, Song G, Zhang H, Xi Y (2015) Targeted mutagenesis in soybean using the CRISPR-Cas9 system. Sci Rep 5:10342. https://doi.org/10.1038/srep10342

Sun Y, Li J, Xia L (2016) Precise genome modification via sequence-specific nucleases-mediated gene targeting for crop improvement. Front Plant Sci 7: 1928. https://doi.org/10.3389/fpls.2016.01928

Wang F, Lin R, Feng J, Chen W, Qiu D, Xu S (2015) TaNAC1 acts as a negative regulator of stripe rust resistance in wheat, enhances susceptibility to Pseudomonas syringae, and promotes lateral root development in transgenic Arabidopsis thaliana. Front Plant Sci 6:108. https://doi.org/10.3389/fpls.2015.00108

Wang F, Wang C, Liu P, Lei C, Hao W, Gao Y, Liu YG, Zhao K (2016) Enhanced Rice blast resistance by CRISPR/Cas9-targeted mutagenesis of the ERF transcription factor gene OsERF922. PLoS One 11(4):e0154027. https://doi.org/10.1371/journal.pone.0154027

Wang Y, Cheng X, Shan Q, Zhang Y, Liu J, Gao C, Qiu JL (2014) Simultaneous editing of three homoeoalleles in hexaploid bread wheat confers heritable resistance to powdery mildew. Nat Biotechnol 32(9):947-951. https://doi.org/10.1038/nbt.2969

Xu RF, Li H, Qin RY, Li J, Qiu CH, Yang YC, Ma H, Li L, Wei PC, Yang JB (2015) Generation of inheritable and "transgene clean" targeted genome-modified rice in later generations using the CRISPR/Cas9 system. Sci Rep 5:11491. https://doi.org/10.1038/srep11491

Yang B, Sugio A, White FF (2006) Os8N3 is a host disease-susceptibility gene for bacterial blight of rice. Proc Natl Acad Sci U S A 103(27):10503-10508. https://doi.org/10.1073/pnas.0604088103

Yang J, Luo D, Yang B, Frommer WB, Eom JS (2018) SWEET11 and 15 as key players in seed filling in rice. New Phytol 218(2):604-615. https://doi.org/10.1111/nph.15004

Young R, Passet B, Vilotte M, Cribiu EP, Beringue V, Le Provost F, Laude H, Vilotte $J L$ (2009) The prion or the related Shadoo protein is required for early mouse embryogenesis. FEBS Lett 583(19):3296-3300. https://doi.org/10.1016/j.febslet.2009.09.027

Yuan M, Chu Z, Li X, Xu C, Wang S (2009) Pathogen-induced expressional loss of function is the key factor in race-specific bacterial resistance conferred by a recessive R gene xa13 in rice. Plant Cell Physiol 50(5):947-955. https://doi.org/10.1093/pcp/pcp046

Yuan M, Chu Z, Li X, Xu C, Wang S (2010) The bacterial pathogen Xanthomonas oryzae overcomes rice defenses by regulating host copper redistribution Plant Cell 22(9):3164-3176. https://doi.org/10.1105/tpc.110.078022
Zhang H, Wang S (2013) Rice versus Xanthomonas oryzae pv. Oryzae: a unique pathosystem. Curr Opin Plant Biol 16(2):188-195. https://doi.org/10.1016/j. pbi.2013.02.008

Zhou H, Liu B, Weeks DP, Spalding MH, Yang B (2014) Large chromosomal deletions and heritable small genetic changes induced by CRISPR/Cas9 in rice. Nucleic Acids Res 42(17):10903-10914. https:/doi.org/10.1093/nar/gku806

\section{Publisher's Note}

Springer Nature remains neutral with regard to jurisdictional claims in published maps and institutional affiliations.

\section{Submit your manuscript to a SpringerOpen ${ }^{\circ}$ journal and benefit from:}

- Convenient online submission

- Rigorous peer review

- Open access: articles freely available online

- High visibility within the field

- Retaining the copyright to your article

Submit your next manuscript at $\boldsymbol{\nabla}$ springeropen.com 